\title{
KPI-linked transformation process for digitized production through the systematic analysis and evaluation of trends
}

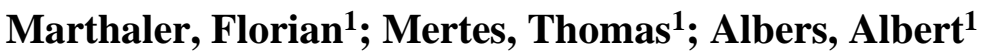 \\ ${ }^{1}$ Karlsruhe Institute of Technology (KIT) \\ IPEK Institute of Product Engineering \\ (Albert.Albers, Florian.Marthaler)@kit.edu \\ uvdlj@student.kit.edu
}

\begin{abstract}
Planning for production is based on a comparison between the target and actual status. Performance figures are used to prioritize and evaluate these. In small companies with limited resources, cost-intensive developments such as setting up production can seriously jeopardize the success of a company. The lack of key figures makes it difficult to prioritize measures. For this reason, a systematic trendbased foresight approach is presented and evaluated in this paper. With the help of systematic foresight for product development, it is possible to prioritize development scopes, identify innovation potential in features and identify transformation stages for an existing production system. In order to evaluate the result, the persons involved in the systematics are interviewed. Three people with varying levels of participation were involved in the implementation, two of whom already have previous knowledge of product development. The created acceptance and trust show that this approach can support in taking future decisions. The identification of product characteristics on the basis of trends leads to a reduction of the uncertainty of future developments and the associated risk, also because developments are classified in several stages. The realization of irrelevant product features is avoided and future relevant features are uncovered. The structured approach of the systematic approach ensures a high level of safety during implementation and can be easily transferred to a production system. It is important to consider that the production system is understood as a product.
\end{abstract}

\section{Keywords: Foresight, strategic planning, production system development}

\section{Introduction}

The selection and resource-efficient design of manufacturing processes, machine tools and associated process chains determine the competitiveness of manufacturing companies and in the future represent a market differentiation feature (Abele \& Reinhart, 2011). In small companies, larger investment activities can lead to significant handicaps and affect competitiveness due to a weaker resource base compared to large companies (Letmathe \& Witt, 2012). The selected time horizon in the strategic planning significantly determines the uncertainty and the possibility of future developments. These uncertainties represent a 
fundamental problem for the planning of relevant tasks and decisions to the company. The use of explorative, projective and recursive methods, which take into account a broad spectrum of possible influences and developments, enables the planning to act in a comprehensible and transparent manner with reduced uncertainty (Behrens, 2003). Strategic planning for production is based on a target/actual comparison and development fields found are evaluated and prioritized by means of performance indicators (KPIs). The scenario technique takes long-term uncertainties into account (Grundig, 2015; Pawellek, 2014; Westkämper \& Löffler, 2016). In a small company with a low use of performance indicators, this prioritisation is made more difficult. For this reason, fields of development should be identified and prioritised for possible future developments. For example, information about the future can be created on the basis of specific scenarios and support the processes of product development in some functions (Albers, Meyer-Schwickerath, \& Siebe, 2012). In a systematic foresight for product development developed by Marthaler et al., product profiles are generated that take into account the influence of future developments and uncertainties and are finally prioritized them into a roadmap for a short, medium and long-term planning horizon (Marthaler, Stehle, Siebe, \& Albers, 2020). As a prerequisite, the production system to be developed must be understood as a product so that the method can be transferred to it. The production is to be transformed in several stages.

\section{State of the art}

In the following, different procedures for the strategic planning of production and product development under consideration of future influences are presented.

\subsection{Strategic planning in production}

The design of the production system as well as the knowledge of technologies is an important success factor for the production of competitive products and a long-term goal of strategic planning. Scenarios are derived to take account of the uncertainties, on the basis of which change measures to achieve the production goals are defined and scheduled in a roadmap (Westkämper \& Löffler, 2016). Future situations and their influencing factors are represented by a scenario. The possible development of the situation is also shown (Gausemeier \& Plass, 2014). A scenario considers time spans between ten and 15 years, while the integration of foresights enables short-term planning with clearly assumed developments. Trends provide a discernible direction for medium-term planning (Fink \& Siebe, 2006). Based on the strategy definition, an analysis of the actual situation of product and production structures will reveal fields of action and measures to change the production system in order to achieve the strategic goals. Tasks and requirements for the product and existing resources in production are identified. The comparison of the requirements with the analysed resources of the existing production system results in change measures for this (Westkämper \& Löffler, 2016). Possible fields of action are manufacturing, procurement, toolmaking, development, assembly, technology and organization (Pawellek, 2014; Westkämper \& Löffler, 2016). The recording of performance figures supports and evaluates the search for potential in the individual fields of action. Scheduling the measures for short, medium and long-term periods serves to achieve the strategic goals (Westkämper \& Löffler, 2016). This underlines the procedure according to Grundig. In order to achieve the objectives, the performance of the company is measured against the goals set or similar companies. The production potential is quantified with performance figures. In the event of deficits and deviations, measures of change are initiated (Grundig, 2015). Pawellek uses the target/actual comparison to divide strategy planning into two variants. In the present oriented variant, measures are derived by the comparison of reference and target state, by unfulfilled requirements. The visionary planning takes a retrospective approach. Target states are defined on the basis of future visions. Based on the 
target states, retrospective measures are sought to achieve the current situation. In order to consider uncertainties in planning, the scenario technique is also used to identify several developments and possible changes and to adjust them when defined events occur. A concretisation based on performance figures makes it possible to prioritise the measures. To implement the strategic measures, a target concept is developed in structural planning, refined in system planning and implemented in the execution phase (Pawellek, 2014).

\subsection{Foresight in the product development}

The 3-cycle model of product development according to Gausemeier and Plass illustrates product development as an interplay between strategic product planning, product development and production system development. With the help of forecasting methods such as Delphi studies, trend analyses and scenario techniques, future success potentials and options for action are identified, on the basis of which a business strategy is developed (Gausemeier \& Plass, 2014). According to Fink and Siebe, foresights are used for short-term, trends for medium-term and scenarios for long-term planning of future product generations (Fink \& Siebe, 2011). The choice of the foresight method depends on the intended planning horizon (Siebe, 2018) and allows the consideration of the future to identify short-, medium- and long-term relevant product features (Marthaler et al., 2019). In a system developed by Martahler et al., the results of the foresight are to be incorporated into the product development process (Marthaler et al., 2020). Generated future product features serve as a link between foresight and product development (Albers, Dumitrescu et al., 2018) and describe necessary differentiation features without specifying a technical solution (Albers, Heitger et al., 2018). The hypothesis of the model of PGE - product generation development, that references are a prerequisite for any development, and Dörner's understanding of the problem serve as the basis for the system. According to Dörner, there is an actual state that must be converted into a defined target state with the support of product development (Albers, Dumitrescu et al., 2018). The use of a reference system influences the development risk and provides advantages to stand out from the competition (Albers, Rapp, Birk, \& Bursac, 2017). The system consists of three modules and comprises seven steps. The reference system is formed in the first module by analyzing and evaluating the product features of the actual state. Future relevant product features are the result of the second module. To identify the features, intuitive and deductive methods are used to derive the results of the prediction. In the third module, the derived product features are evaluated using possible product scenarios and presented in a cross-generational roadmap. The evaluation and subsequent classification of the features into a short, medium and long-term product generation is based on future robustness and the need for change. The conformity of product features regarding to the individual product scenarios is summarized in future robustness. This means: the more relevant a feature is in the foresight, the greater the value of future robustness. The need for change indicates the satisfaction of the customers of the product scenarios with regard to a product feature and the resulting need for change (see figure 1). In the fourth step, product scenarios are created, which are used in the fifth step to evaluate the updated product features according to future robustness and need for change. The need for change and future robustness are visualized in a diagram in the sixth step. This is divided into four quadrants, which correspond to a short, medium and long-term variation or no variation at all. Depending on the value of the need for change and future robustness, the product feature is assigned to a point of variation and thus prioritized. With the support of the generated features and variation points, specific development orders can be transferred into a roadmap. The product development process is concluded with the validation of the new product profiles at (sub)system level (Marthaler et al., 2019). 


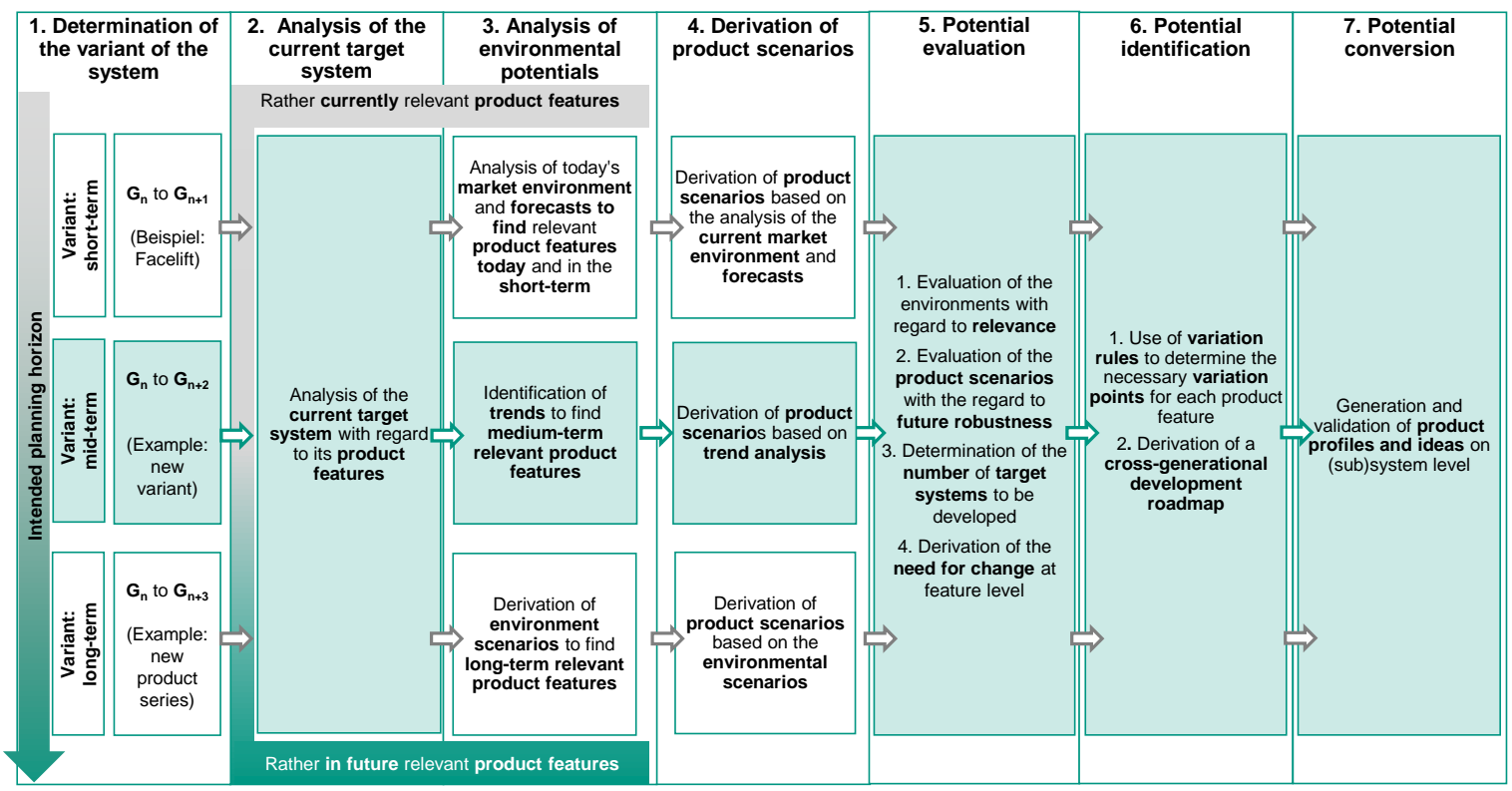

Figure 1. Systematic foresight for product generation development (Marthaler et al., 2019)

\section{Research methodology}

The state of research shows that strategic actions in the development of production systems are based on a comparison between actual and target states. The identified development fields are prioritized based on existing performance figures.

In particular, the competitiveness of small businesses is influenced by larger investments. Due to a weaker resource base compared to large companies, risky decisions can have considerable disadvantages and seriously jeopardize the success of a company (Letmathe \& Witt, 2012). The expansion and the development of a production represent such a critical decision due to high acquisition costs of machines (Westkämper \& Löffler, 2016). Uncertainties and a lower number of key figures make prioritisation difficult. The objective of this article is therefore to use a systematic approach to strategically plan the production system of an expanding small company taking into account trends, in order to minimize the economic risk by prioritizing the identified fields of development. The following questions will be answered:

- How can the use for cross-generational planning and prioritization of a production system be successful? What does such an approach look like, for exemplary purposes, in the production system of an expanding small company?

- What contribution does the system make to strategic planning in a small company?

The systematic foresight on product generation development according to Marthaler et al. is applied to the existing production system of a cooperating small company within the framework of a research project. The production system is regarded as a product. The development of the production system is planned in order to satisfy an increasing demand. The produced quantities of a critical assembly serve as KPI and correspond to the strategic target planning. Existing performance figures and performance measurement systems are ignored. The prioritization of the development fields is carried out with the analysis of trends that result in short, medium and long-term transformation stages for the production system. An evaluation based on a questionnaire is intended to illustrate the contribution to strategic planning. The questionnaire was developed by Marthaler et al. and includes the key factors for the successful application of systematic foresight (Marthaler et al., 2019). All persons involved are interviewed. The generated fields of development serve only for orientation and possible recommendations for 
action to support strategic planning. For reasons of confidentiality, the results are presented in a reduced form.

\section{Results}

In the first part of the following chapter, the application of foresight in product development according to Marthaler et al. to the existing production system is illustrated. The second part includes the evaluation.

\subsection{Application of the systematic foresight}

\subsubsection{Determination of the variant of the system}

Small businesses mainly make short to medium-term decisions. For this reason, the system is used for a medium-term planning horizon. For this purpose, the steps highlighted in green in Figure 1 are carried out.

\subsubsection{Analysis of the current target system}

In this step, the current target system, which serves as a reference system, is determined. The product features are analyzed and evaluated with a Likert scale $(++,+, 0,-,--)$. As reference system or actual state, the current production system is selected, which is to be further developed in order to achieve the specified target values. The features of the production system are identified by characteristics from literature and a preceding process analysis and then evaluated by the strategic management according to degree of fulfillment and relevance for the company. In Figure 2, the analysed features of the production system are evaluated according to relevance in the column highlighted in blue.

\subsubsection{Analysis of the environmental potentials}

In the following step, future product properties are uncovered by comparing the properties with the results of the foresight.

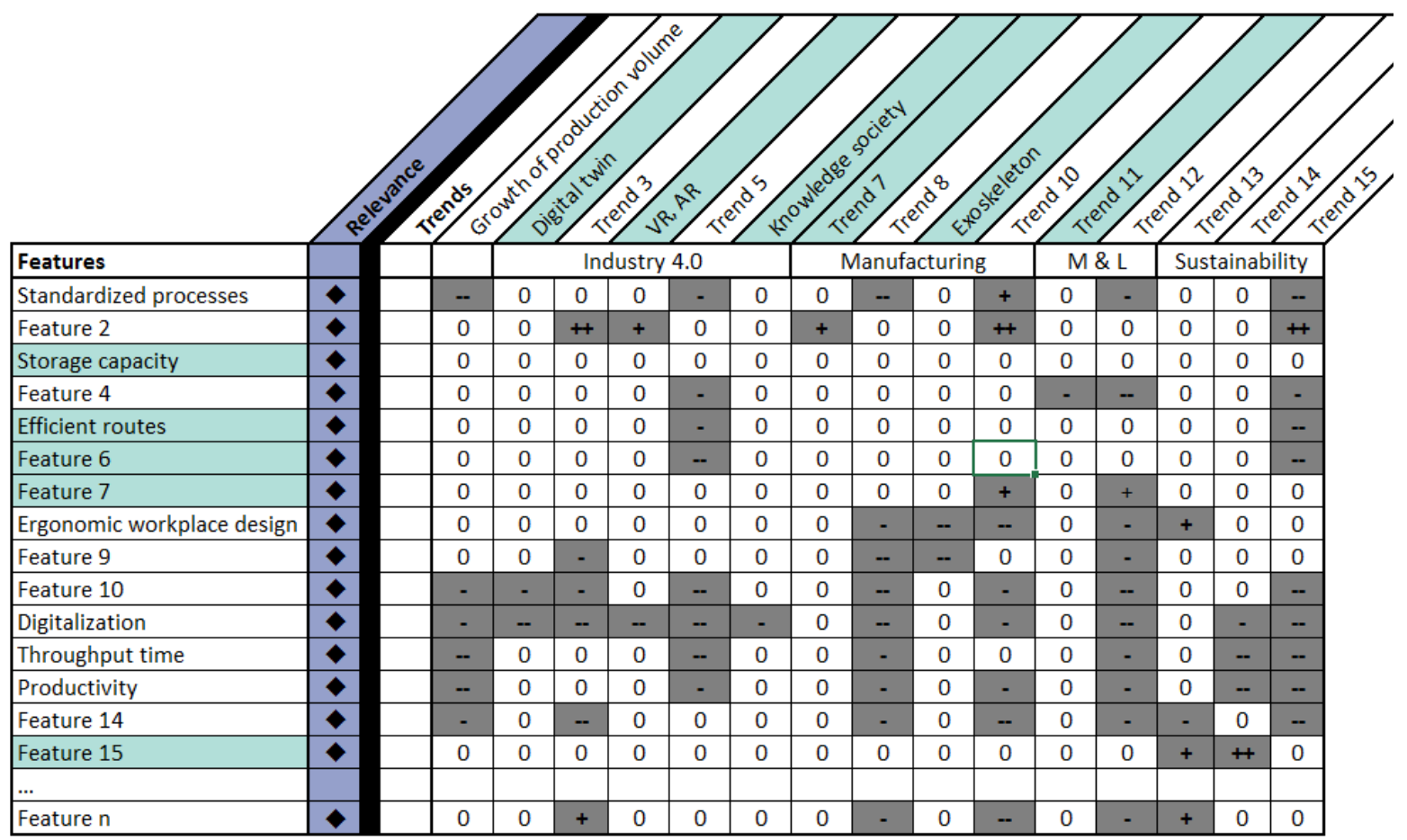

Figure 2. Relevance evaluation and consistency analysis between identified trends with product features to find future relevant product features 
To carry out the trend analysis, contributions to the problem are compiled from journals, current literature and company goals. The identified trends are assigned to superordinate categories and redundancies are summarized. Figure 2 shows a section of the consistency analysis of product features and trends for the analysis of the environmental potentials. The top row shows the resulting trends from industry 4.0, production and manufacturing, mobility and logistics ( $\mathrm{M} \&$ L) and sustainability.

Then, consistency analysis is used to update the features of the production system. For this purpose, trends and features are examined for independence, direct and indirect consistency in the form of a strong or weak expression $(++,+, 0,-,--)$. Existing consistencies between trend and product feature are highlighted in grey. From independent features and trends, white spots and irrelevant features can be identified. This case is marked by a "0" in the matrix of Figure 2. Irrelevant features of the reference system are identified by lines with missing dependencies and marked in green. According to this, the features storage capacity, efficient routes, feature 6, 7 and 15 are to be classified as irrelevant and removed. New product features are generated from the columns or trends that have a low consistency. These are also highlighted in green. The product feature of the optical assistance systems is derived from the trend of virtual and augmented reality. Further generated features are listed in Table 1.

Table 1. Generated future product features of independent trends

\begin{tabular}{|l|l|}
\hline Trend & Generated product feature \\
\hline Digital twin & Process data \\
\hline Virtual and augmented reality & Optical assistance systems \\
\hline Knowledge society & Introduction Wiki \\
\hline Exoskeleton & New feature \\
\hline$\ldots$ & $\ldots$ \\
\hline Trend n & New feature \\
\hline
\end{tabular}

\subsubsection{Derivation of product scenarios}

To carry out the potential assessment in the next step, several product scenarios are created and evaluated according to the probability of occurrence. Using the pairwise comparison shown in Figure 3, six scenarios are prioritised.

\begin{tabular}{|c|c|c|c|c|c|c|c|c|}
\hline & \multirow{2}{*}{$\begin{array}{l}\text { Strategic } \\
\text { planning }\end{array}$} & \multirow{2}{*}{$\begin{array}{l}\text { Employee } \\
\text { production }\end{array}$} & \multicolumn{3}{|c|}{ Magic triangle of production } & \multirow{2}{*}{$\begin{array}{l}\text { Economic } \\
\text { recession }\end{array}$} & \multirow{2}{*}{ Total } & \multirow{2}{*}{$\begin{array}{l}\text { Percentage } \\
\text { share }\end{array}$} \\
\hline & & & Costs & Time & Quality & & & \\
\hline Strategic planning & & 2 & 2 & 2 & 2 & 2 & 10 & 33,33 \\
\hline Employee production & 0 & & 2 & 1 & 1 & 2 & 6 & 20,00 \\
\hline Costs & 0 & 0 & & 0 & 1 & 2 & 3 & 10,00 \\
\hline Time & 0 & 1 & 2 & & 2 & 2 & 7 & 23,33 \\
\hline Quality & 0 & 1 & 1 & 0 & & 2 & 4 & 13,33 \\
\hline Economic recession & 0 & 0 & 0 & 0 & 0 & & 0 & 0,00 \\
\hline & & & \multicolumn{4}{|c|}{ Legend } & & \\
\hline & & & \multicolumn{4}{|c|}{$\begin{array}{l}2: 0=1 \text { st scenario more imprtant than } 2 \text { nd scenario } \\
1: 1=1 \text { st scenario as important as } 2 \text { nd scenario } \\
0: 2=1 \text { st scenario less important than } 2 \text { nd scenario }\end{array}$} & & \\
\hline
\end{tabular}

Figure 3. Prioritization of derived product scenarios

The scenario of management and strategic planning has the highest priority with $33.33 \%$. The employees in production who are in direct contact with the developed production system are weighted at $20 \%$. Further scenarios form the conflicting goals of production: costs $10 \%$, time 
$23.33 \%$ and quality $13.33 \%$. A defined production quantity with low manufacturing costs, high product quality and short lead times are required. The economic recession, which represents a negative scenario, can be rejected by comparison with a probability of $0 \%$.

\subsubsection{Potential evaluation}
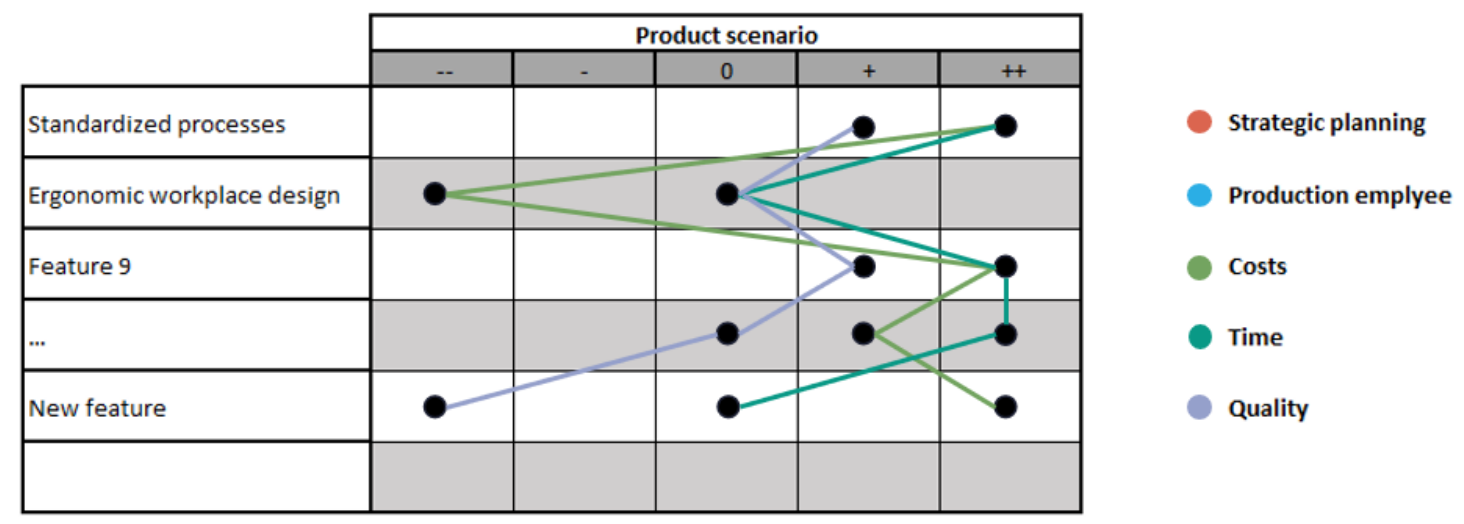

Figure 4. Future robustness of the properties with regard to the product scenarios

In the fifth step of the systematic foresight, the features of the production system are evaluated by the scenarios of the previous step with regard to the need for change and future robustness. The future robustness of the individual scenarios, with the exception of the strategic scenario and the production employees, is shown in Figure 4. A high future robustness corresponds to a high relevance of the property in the shown scenario. The need for change is evaluated analogously to future robustness. A high value of the need for change corresponds to low satisfaction and requires a quick variation of the product feature. The weighting of the individual scenarios from step 4 is also included in the evaluation. For this reason, the excluded negative scenario is not listed. The evaluation of the potential evaluation is carried out with the help of a software tool developed by Marthaler et al. (2019). The result, a feature variation portfolio, is shown in Figure 4 and explained in step 6.

\subsubsection{Potential identification}

The evaluation of the features by the scenarios leads to the feature variation portfolio shown in Figure 5. The division into four quadrants prioritizes the developments of the product features and concretizes their points of variation. The classification is based on the mean values of future robustness and the need for change of the properties. The $\mathrm{x}$-axis indicates the future robustness and the $y$-axis the need for change. The first quadrant stands for a short-term variation and represents features that require a high degree of future robustness and a high need for change and thus measures to achieve short-term production targets. The second quadrant stands for a medium-term variation with a high need for change and medium robustness. The third quadrant illustrates the scope of development in the product features for a long-term planning horizon. Characteristics in the fourth quadrant do not have to be varied.

The KPIs are mapped to the variation points in time so that short-term measures can be implemented to secure the first production target. With increasing production targets, the performance figures are assigned to the medium-term or long-term variation. In order to achieve the first production goal, for example, the development of features such as digitization, productivity, standardized processes, etc. is recommended. In contrast to the procedure and in order to ensure the achievement of the short-term goals, the division of the quadrants is 
additionally adapted in order to concentrate developments on essential features and their implementation (cf. green boundary lines). Resulting product profiles and interactions of the characteristics are derived in the next step.

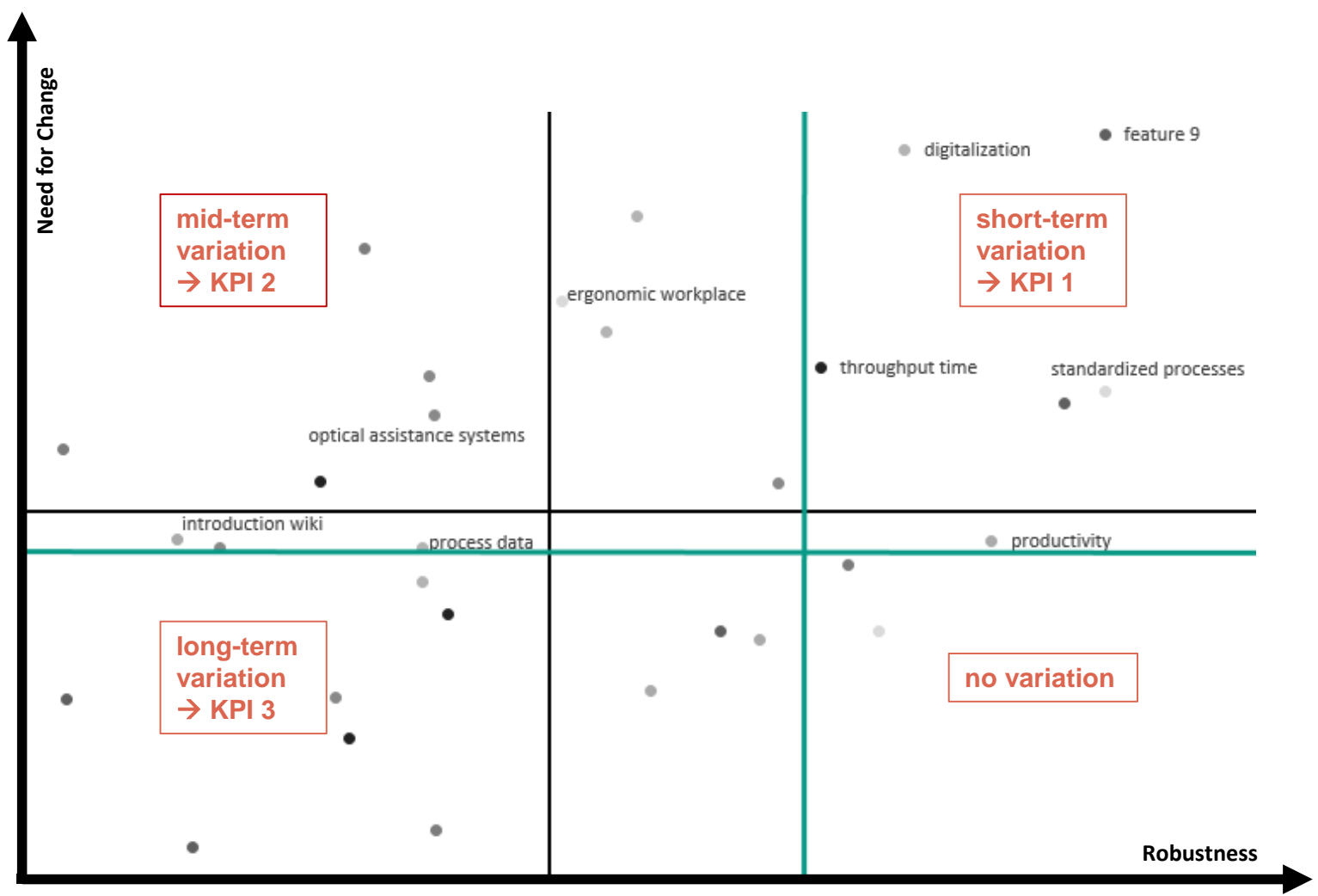

Figure 5. Generated feature variation portfolio for the visualization of the variation times of the product features on the basis of future robustness and need for change

\subsubsection{Potential conversion}

Figure 5 shows the variation times for the development scopes of the individual transformation stages of the production system. The following step is limited to the short-term variation and its interactions and is explained exemplarily. The product profile is characterized, for example, by standardized processes, digitization and throughput time. Standardized processes are a core element of a production system and ensure transparency and improved coordination. The process is optimised and complexity reduced by the clear nature of the instructions. The benefits of standardized processes can be seen in reduced non-productive times, increased process reliability, faster training of employees and the associated more economical production of the product. It is also a prerequisite for mass production. The reduction non-productive times results in a shorter throughput time of the products. The productivity can be increased many times over. The establishment of possible communication interfaces represents measures with regard to digitization and serves as a basis for the next transformation stages.

The fields of development of the production system presented here result in higher productivity and make a major contribution to the achievement of objectives. However, there are interactions with other subsystems. For example, it should be noted that with the introduction of further automated systems, personnel must be hired for operation and maintenance. Increased production figures can lead to new bottlenecks in the production process, for example in quality assurance, assembly or suppliers. The integration of fixed partners reduces the risk of bottlenecks in material procurement. 
The second product generation or transformation phase of the production system prioritizes the medium-term developments. An introduction of optical assistance systems and further optimizations of the ergonomics of the workplaces support the employees. These measures lead to an increase in productivity. On the basis of the first measures regarding digitization of the short-term transformation stage, process data, for example, will be mapped and a wiki introduced in the medium-term transformation.

\subsection{Evaluation of the system}

With the help of systematic foresight for product development, it is possible to prioritize development scopes, identify innovation potential in features and identify transformation stages for an existing production system. In order to evaluate the result, the persons involved in the systematics are interviewed. Three people with varying levels of participation were involved in the implementation, two of whom already have previous knowledge of product development. For evaluation purposes, the key factors identified according to Marthaler et al. for a generationspanning foresight are evaluated with regard to the degree of fulfillment $(1=$ not fulfilled, $5=$ fully fulfilled) (Marthaler et al., 2019). The results are shown in Figure 6. The green line summarizes the individual evaluation results.

The results of the evaluation show that a high degree of trust (M1.2) and acceptance (M1.1) in the systematic foresight can be created among all participants. This also applies to people who have no previous knowledge of product development. The easy integration of internal customers, for example through targets and company-relevant trends (P1) and a look into the future of product developers (S1) stimulates discussion about today's needs (P3). The steps are structured in a comprehensible way (M4), which favours the transfer to a production system, but the application is not intuitively possible (M3). One possible cause is the execution of the consistency analysis. When subjectively assessing the consistency between trend and product characteristic, one's own decision is occasionally questioned as to whether existing dependencies have been correctly identified. The high number of trends and product features, as well as the consistency analysis, require a lot of time to complete the third step. The advantages can well justify the initial effort. The use of software to generate the property variation portfolio in the sixth step reduces the effort during implementation. Another software tool or consistency analysis approach can support intuitive use.

Overall, according to Marthaler et al., many of the key factors identified are well met by the systematic foresight (Marthaler et al., 2019). The integration of corporate competencies (M8), the comparison with completed development projects (M9) and the identification of possible competitive strategies can be expanded. The key factor (S3) needs to be revised to understand it. 


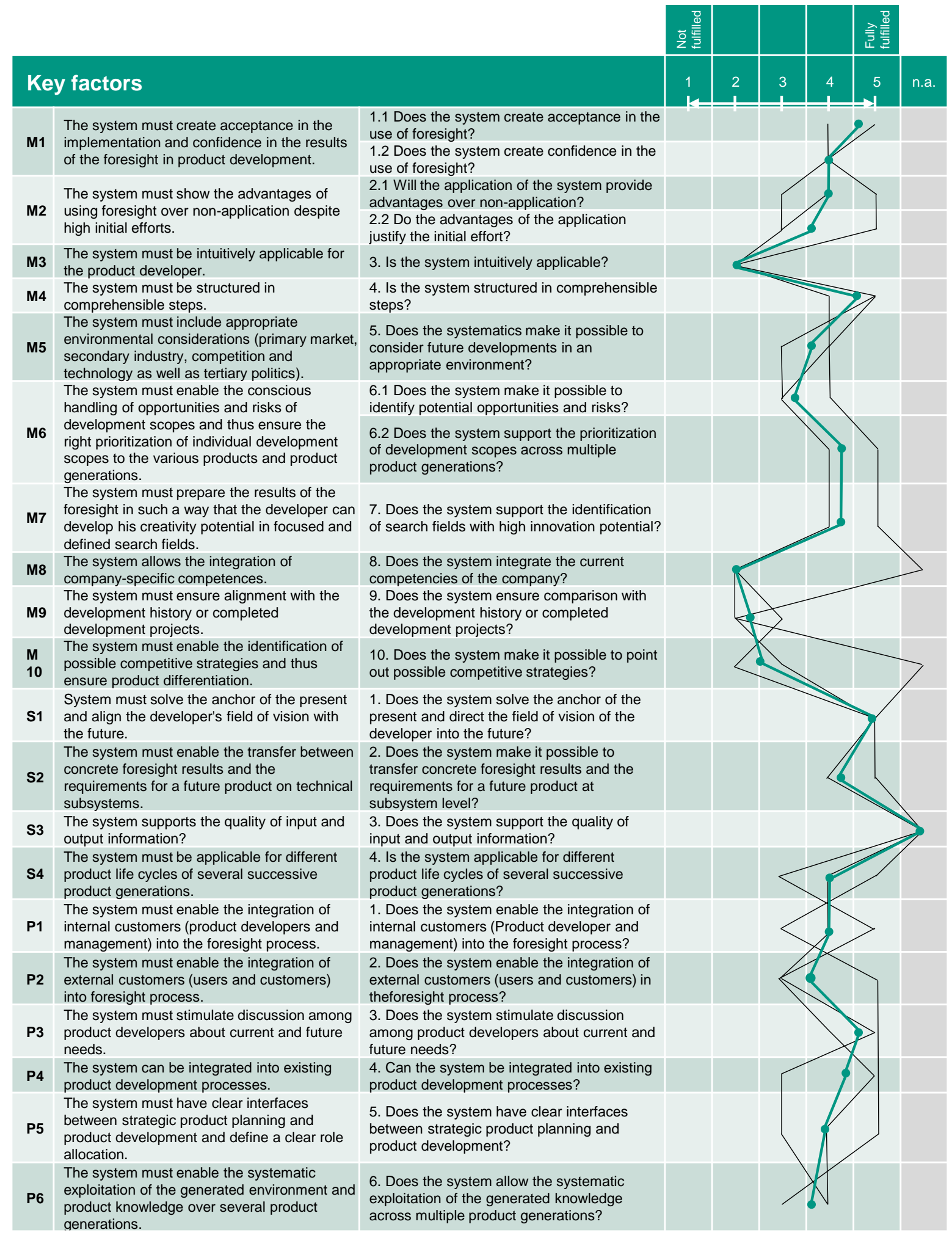

Figure 6. Evaluation results on the application of systematic foresight to a production system (Marthaler et al., 2019)

\section{Interpretation and outlook}

The created acceptance and trust show that the systematic foresight can support future decisions and make a helpful contribution. The elimination and generation of product characteristics on the basis of trends leads to a reduction of the uncertainty of future developments and the 
associated risk, also because developments are classified in several stages. The realization of irrelevant product features is avoided and future relevant features are uncovered. The structured approach of the system ensures a high level of safety during implementation and can be easily transferred to a production system. It is important to consider that the production system is understood as a product. The subjective analysis of the identified trends and the subsequent consistency analysis leaves room for speculation and misinterpretations during application. It is often not clear to how far the features and trends are consistent and whether they are strong or weak. To eliminate or generate the product characteristics, independence is essential. For this systematic step, a classification into "consistent" and "independent" would be sufficient. An appropriate size of the development team can facilitate consistency analysis and verify identified trends. This also applies to the derivation and evaluation of product scenarios. The classification of characteristics in the variation portfolio according to the average values of future robustness and the need for change results in many characteristics being recommended for short-term variation. Using an individual concentration or a limitation of the characteristics for a short-term variation, the development can be concentrated on the essential and moves within a manageable framework. Product features can also exceed the variation limits in their development. For example, the feature "digitalization" reveals a very high development potential and a whole implementation is not realizable in one transformation stage. In order to prioritize this potential, it is advisable to reapply the systems limited to this feature, which makes a possible fractal character of the system clear. The handling of dependent features, which are very contrary in their prioritization, must be defined. For example, the introduction of a wiki is recommended at the time of process standardization. Linking the KPIs to the points in time of variation shows the strategic planning possible measures for the respective target value in order to achieve them and supports a more structured development of the production system in short, medium and long-term transformation stages. Particularly in small businesses, the use of such a system can prove helpful in many areas. A final success must be determined by validating the implementation in the company.

\section{References}

Abele, E., \& Reinhart, G. (2011). Zukunft der Produktion. München: Carl Hanser Verlag GmbH \& Co. KG. https://doi.org/10.3139/9783446428058

Albers, A., Dumitrescu, R., Marthaler, F. [F.], Albers, A. A., Kühfuss, D. [D.], Strauch, M. [M.], ... Bursac, N. [N.] (Eds.) (2018). PGE-Produktgenerationsentwicklung und Zukunftsvorausschau: Eine systematische Betrachtung zur Ermittlung der Zusammenhänge.

Albers, A., Heitger, N., Haug, F., Fahl, J., Hirschter, T., \& Bursac, N. [N.] (Eds.) (2018). Supporting Potential Innovation in the Early Phase of PGE - Product Generation Engineering: Structuring the Development of the Initial System of Objectives.

Albers, A., Meyer-Schwickerath, B., \& Siebe, A. [A.] (Eds.) (2012). Integrated use of scenario planning and strategic early warning systems to support product engineering processes.

Albers, A., Rapp, S., Birk, C., \& Bursac, N. [N.] (Eds.) (2017). Die Frühe Phase der Produktgenerationsentwicklung.

Behrens, S. (2003). Möglichkeiten der Unterstützung von strategischer Geschäftsfeldplanung und Technologieplanung durch Roadmapping. Berlin: Logos.

Fink, A., \& Siebe, A. [Andreas] (2006). Handbuch Zukunftsmanagement: Werkzeuge der strategischen Planung und Früherkennung. Campus Management. Frankfurt am Main: Campus. 
Fink, A., \& Siebe, A. [Andreas] (2011). Handbuch Zukunftsmanagement: Werkzeuge der strategischen Planung und Früherkennung (2., aktualisierte und erw. Aufl.). Frankfurt am Main: Campus.

Gausemeier, J., \& Plass, C. (2014). Zukunftsorientierte Unternehmensgestaltung. München: Carl Hanser Verlag GmbH \& Co. KG. https://doi.org/10.3139/9783446438422

Grundig, C.-G. (2015). Fabrikplanung: Planungssystematik - Methoden - Anwendungen (5., aktualisierte Aufl.). München: Hanser.

Letmathe, P., \& Witt, P. (2012). Management von kleinen und mittleren Unternehmen. Zeitschrift Für Betriebswirtschaft, 82(S3), 1-3. https://doi.org/10.1007/s11573-012-0569-9

Marthaler, F. [Florian], Orsolani Uhlig, E., Marthaler, P., Kühfuss, D. [Dennis], Strauch, M. [Markus], Siebe, A. [Andreas], ... Albers, A. [Albert] (Eds.) (2019). Strategische Potentialfindung zur generationsübergreifenden Produktentwicklung mit langfristigem Zeithorizont: Eine qualitative Studie im Live-Lab IP - Integrierte Produktentwicklung.

Marthaler, F. [Florian], Stehle, S., Siebe, A. [Andreas], \& Albers, A. [Albert] (Eds.) (2020). Futue - oriented product engineering through environment scenario by using the example of future forms of mobility in urban living spaces.

Pawellek, G. (2014). Ganzheitliche Fabrikplanung. Berlin, Heidelberg: Springer Berlin Heidelberg. https://doi.org/10.1007/978-3-662-43728-5

Siebe (2018). Die Zukunft vorausdenken und gestalten: Springer Berlin Heidelberg.

Westkämper, E., \& Löffler, C. (2016). Strategien der Produktion. Berlin, Heidelberg: Springer Berlin Heidelberg. https://doi.org/10.1007/978-3-662-48914-7 